\title{
Uso das práticas integrativas e complementares no cuidado com os idosos na atenção primária de saúde: protocolo de scoping review
}

Use of integrative and complementary practices in elderly care in primary health care: scoping review protocol

Uso de prácticas integradoras y complementarias en la atención de personas mayores en la atención primaria de salud: protocolo de scoping review

Recebido: 02/12/2021 | Revisado: 07/12/2021 | Aceito: 11/12/2021 | Publicado: 20/12/2021

Ana Carolina de Avelar Maciel ORCID: https://orcid.org/0000-0002-6358-5742 Universidade Federal de São João del-Rei, Brasil E-mail:anaavelrmaciel@gmail.com Beatriz Felix Santos ORCID: https://orcid.org/0000-0002-7708-4586 Universidade Federal de São João del-Rei, Brasil E-mail:biafelixap@gmail.com

Mayara Ingra dos Santos Leite ORCID: https://orcid.org/0000-0001-9860-9372 Universidade Federal de São João del-Rei, Brasil E-mail:mayaraingra@yahoo.com.br

Amanda Damasceno de Souza ORCID: https://orcid.org/0000-0001-6859-4333 Hospital Felício Rocho, Brasil E-mail: amandas81@gmail.com

Maria Marta Amancio Amorim ORCID: https://orcid.org/0000-0001-8268-2508 Centro de Estudos em Migrações e Relações Interculturais de Lisboa, Portugal E-mail: martamorim@hotmail.com

Aline Medeiros ORCID:https://orcid.org/0000-0001-7486-3245 Universidade Federal de São João del-Rei, Brasil

E-mail: alinemedeirosnutri@hotmail.com

Fernanda Moura Lanza

ORCID: https://orcid.org/0000-0001-8250-180X Universidade Federal de São João del-Rei, Brasil E-mail: fernandalanza@ufsj.edu.br

\section{Resumo}

O objetivo desse protocolo scoping review é identificar e sintetizar as evidências científicas produzidas sobre o uso das Práticas Integrativas e Complementares em Saúde (PICS) por idosos nos serviços de atenção primária à saúde (APS). A revisão seguirá o método proposto pelo Joanna Briggs Institute, que estabelece cinco etapas: 1) identificação da questão de pesquisa; 2) identificação dos estudos relevantes; 3) seleção dos estudos; 4) análise dos dados; e 5) agrupamento, síntese e apresentação dos dados. Para a formulação da pergunta de pesquisa utilizou-se a estratégia Participants (P): idosos; Concept (C): Práticas Integrativas e Complementares em Saúde; Context (C): Atenção Primária à Saúde. Assim a pergunta de pesquisa formulada contemplou o PCC (Participants- ConceptContext): Quais as evidências científicas, no contexto da APS (P), do uso das PICS (C) por idosos (P) para a promoção, manutenção e recuperação da saúde, e para a prevenção de agravos? Os descritores controlados foram selecionados a partir dos Descritores em Ciências da Saúde (DeCS), Medical Subject Headings Section (MeSH), EMTREE e CINAHL Headings. Espera-se que essa scoping review forneça um mapeamento da utilização das PICS por idosos, como método para a promoção da saúde, prevenção de agravos, além da manutenção e recuperação da saúde e, com isso, proporcionar o envelhecimento saudável e com qualidade, a fim de fornecer a atuação da APS como porta de entrada do Sistema Único de Saúde com grande capacidade resolutiva.

Palavras-chave: Literatura de revisão como assunto; Protocolo de pesquisa; Práticas integrativas e complementares; Idoso; Atenção Primária à Saúde.

\footnotetext{
Abstract

The objective of this scoping review protocol is to identify and synthesize the scientific evidence produced on the use of Integrative and Complementary Health Practices (PICS) by the elderly in primary health care services (PHC). The
} 
review will follow the method proposed by the Joanna Briggs Institute, which establishes five steps: 1) identification of the research question; 2) identification of relevant studies; 3) selection of studies; 4) data analysis; and 5) grouping, synthesis and presentation of data. To formulate the research question, the Participants $(\mathrm{P})$ strategy was used: elderly; Concept (C): Integrative and Complementary Practices in Health; Context (C): Primary Health Care. Thus, the research question formulated included the PCC (Participants-Concept-Context): What is the scientific evidence, in the context of PHC (P), of the use of PICS (C) by the elderly (P) for the promotion, maintenance and recovery of health, and for the prevention of injuries? Controlled descriptors were selected from the Health Sciences Descriptors (DeCS), Medical Subject Headings Section (MeSH), EMTREE and CINAHL Headings. It is expected that this scoping review will provide a mapping of the use of PICS by the elderly, as a method for health promotion, disease prevention, in addition to the maintenance and recovery of health and, therefore, providing healthy and quality aging, in order to provide the performance of the PHC as a gateway to the Unified Health System with great resolving capacity.

Keywords: Review literature as topic; Protocols; Complementary therapies; Aged; Primary Health Care.

\section{Resumen}

El propósito de este protocolo de revisión de alcance es identificar y sintetizar la evidencia científica producida sobre el uso de Prácticas de Salud Integrativas y Complementarias (PICS) por los ancianos en los servicios de atención primaria de salud (APS). La revisión seguirá el método propuesto por el Instituto Joanna Briggs, que establece cinco pasos: 1) identificación de la pregunta de investigación; 2) identificación de estudios relevantes; 3) selección de estudios; 4) análisis de datos; y 5) agrupación, síntesis y presentación de datos. Para formular la pregunta de investigación se utilizó la estrategia Participantes (P): anciano; Concepto (C): Prácticas integradoras y complementarias en salud; Contexto (C): Atención Primaria de Salud. Así, la pregunta de investigación formulada incluyó el PCC (Participantes-Concepto-Contexto): ¿Cuál es la evidencia científica, en el contexto de la APS (P), del uso de PICS $(\mathrm{C})$ por ancianos $(\mathrm{P})$ para la promoción, mantenimiento y recuperación de la salud, y para la prevención de lesiones? Los descriptores controlados se seleccionaron de los descriptores de ciencias de la salud (DeCS), la sección de títulos de materias médicas (MeSH), los títulos EMTREE y CINAHL. Se espera que esta revisión de alcance proporcione un mapeo del uso de PICS por las personas mayores, como método de promoción de la salud, prevención de enfermedades, además del mantenimiento y recuperación de la salud y, por lo tanto, proporcionando un envejecimiento saludable y de calidad, en con el fin de brindar la actuación de la APS como puerta de entrada al Sistema Único de Salud con gran capacidad resolutiva.

Palabras clave: Literatura de revisión como asunto; Protocolos; Terapias complementarias; Anciano; Atención Primaria de Salud.

\section{Introdução}

Instituído em 1988, com a promulgação da Constituição Cidadã, o Sistema Único de Saúde (SUS) garante acesso integral, universal e gratuito ao sistema público de saúde para toda a população brasileira sem discriminação (Brasil, 1988). A implementação do SUS representa a democratização da saúde e uma mudança na lógica da atenção à saúde que passou de um quadro reduzido ao tratamento de enfermidades para ações de promoção da saúde, prevenção de agravos e recuperação da saúde (Giovanella et al., 2019).

Como porta preferencial de entrada do usuário no SUS tem-se a Atenção Primária à Saúde (APS), que é o centro de comunicação com todos os serviços da Rede de Atenção (Nascimento et al., 2020). A APS é caracterizada por "um conjunto de ações de saúde, no âmbito individual e coletivo, que abrange a promoção e a proteção da saúde, a prevenção de agravos, o diagnóstico, o tratamento, a reabilitação, a redução de danos e a manutenção da saúde que visam o desenvolvimento de uma atenção integral que impacte de maneira positiva a situação de saúde das coletividades” (Brasil, 2017a, sp).

No território brasileiro, a APS acontece com o mais alto grau de descentralização e capilaridade tendo a Estratégia Saúde da Família (ESF) como uma de suas diversas estratégias governamentais para "expansão, qualificação e consolidação" da APS (Brasil, 2017a). A ESF é responsável por estabelecer um vínculo entre os profissionais de saúde e a população, obter conhecimento da realidade das famílias pelas quais é responsável, detectar os problemas de saúde predominantes e situações de risco às quais a população em seu território de abrangência está exposta para fornecer assistência integral (Brasil, 2017a). Constitui-se assim como um local com grande potencial de atuação às Práticas Integrativas e Complementares em Saúde (PICS) (Habimorad et al., 2020; Silva et al., 2020). 
As PICS “são recursos terapêuticos que buscam a prevenção de doenças e a recuperação da saúde, com ênfase na escuta acolhedora, no desenvolvimento do vínculo terapêutico e na integração do ser humano com o meio ambiente e a sociedade" (Brasil, 2021, sp), contribuindo nas ações de promoção à saúde e, consequentemente, na mudança do modelo biomédico (Brasil, 2018a) que ainda é considerado predominante em certas localidades (Silva et al., 2019).

As PICS denominadas pela Word Health Organization (WHO) de medicina complementar/ alternativa (WHO, 2019), constituem práticas e recursos terapêuticos cuja implementação visa a utilização de mecanismos naturais usados para a prevenção de agravos e recuperação da saúde (Pinto et al., 2020). Essas PICS atuam fortalecendo os princípios e diretrizes do SUS (Brasil, 2006a; Moebus \& Merhy, 2017), promovendo o cuidado em saúde com ênfase na integralidade da assistência, estabelecendo uma atenção voltada ao estilo de vida, estado emocional e espiritual de cada pessoa objetivando oferecer uma assistência completa e humanizada (Pinto et al., 2020).

Elas foram institucionalizadas no SUS por meio da Política Nacional de Práticas Integrativas e Complementares (PNPIC) nos anos de 2006 (Brasil, 2006b), 2017 (Brasil, 2017b) e 2018 (Brasil, 2018b). Atualmente são 29 PICS que são disponibilizadas na rede de atenção do SUS, sendo elas: Medicina Tradicional Chinesa/Acupuntura, Medicina Antroposófica, Homeopatia, Plantas Medicinais e Fitoterapia, Termalismo Social/Crenoterapia, Arteterapia, Ayurveda, Biodança, Dança Circular, Meditação, Musicoterapia, Naturopatia, Osteopatia, Quiropraxia, Reflexoterapia, Reiki, Shantala, Terapia Comunitária Integrativa, Yoga, Apiterapia, Aromaterapia, Bioenergética, Constelação familiar, Cromoterapia, Geoterapia, Hipnoterapia, Imposição de mãos, Ozonioterapia e Terapia de Florais (Brasil, 2021).

As PICS também estão interligadas com a Política Nacional de Promoção da Saúde, uma vez que a promoção da saúde envolve "um conjunto de ideias e práticas que compreendem o processo saúde-doença e seus determinantes, e permitem a articulação de saberes técnicos e populares juntamente com a mobilização de recursos institucionais e comunitários, públicos e privados para seu enfrentamento e resolução" (Brasil, 2018 a,b,c).

Mediante a transição demográfica que está ocorrendo na população brasileira, há uma necessidade de promoção e de criação de políticas visando o incentivo ao bem-estar individual e coletivo da população idosa. A implementação de mecanismos capazes de reduzir os impactos psíquicos, sociais e físicos que estão presentes na vida do idoso e dos que estão no processo de transição da juventude para a terceira idade se faz necessário. Diante desse cenário, compreendemos que essa população precisa de uma atenção focada na prevenção, promoção e reabilitação mais específicas para seu processo de senilidade e/ou senescência (Almeida et al., 2021).

Estudo realizado por Marques, Francisco, Bacuru, Rodrigues, Malta \& Barros (2020) com base em dados da Pesquisa Nacional de Saúde de 2013 demonstrou que o uso das PICS foi referido por apenas 5,4\% dos idosos e, dentre esses, a maioria relatou o uso de plantas medicinais e fitoterapia (62,6\%), seguida por acupuntura $(22,2 \%)$ e homeopatia (11,2\%). Em relação ao uso de PICS por idosos com a presença de doenças crônicas, foram observados nesse estudo maiores proporções de uso de PIC entre os idosos com hipercolesterolemia, artrite ou reumatismo, problema crônico de coluna e depressão.

Nessa perspectiva, tendo em vista o crescente processo de envelhecimento da população, observa-se a necessidade em se efetivar a integralidade da assistência no cotidiano da APS para o fortalecimento do SUS (Nascimento et al.,). Nascimento et al., 2020), em especial, nos “campos da prevenção de agravos e da promoção, manutenção e recuperação da saúde” (Brasil, 2006a, p.4). Considerando o potencial da APS para atuação longitudinal propiciado sobretudo pela ESF, compreende-se que esse é o local propício para a inclusão das PICS no cardápio de ações da APS e para o seu desenvolvimento em caráter multiprofissional (Brasil, 2018c).

Dessa forma, nessa scoping review questiona-se quais são as evidências científicas produzidas sobre o uso das PICS por idosos nos serviços de APS para a promoção, manutenção e recuperação da saúde, e para a prevenção de agravos?

Assim o objetivo desse protocolo scoping review é identificar e sintetizar as evidências científicas produzidas sobre o 
uso das PICS por idosos nos serviços de APS.

\section{Metodologia}

Para encontrar a resposta para a questão do estudo, optou-se por realizar uma scoping review ou revisão de escopo por ser um método que fornece uma visão geral das evidências de pesquisa existentes, mas sem gerar uma resposta sintetizada para uma pergunta de pesquisa diferente (Sucharew \& Macaluso, 2019).

O objetivo da scoping review é realizar o mapeamento da literatura sobre determinado tema em que revisões ainda não foram publicadas. Esse tipo de revisão é adequado a tópicos amplos, podendo reunir vários desenhos de estudos e tendo como finalidade reunir os vários tipos de evidências e mostrar como foram produzidas (Cordeiro \& Soares, 2019).

A revisão seguirá o método proposto pelo Joanna Briggs Institute (JBI), Reviewers Manual 2020 (Aromataris \& Munn, 2020), que estabelece cinco etapas 1) identificação da questão de pesquisa; 2) identificação dos estudos relevantes; 3) seleção dos estudos; 4) análise dos dados; e 5) agrupamento, síntese e apresentação dos dados. Essas etapas serão detalhadas na próxima seção.

\section{Resultados}

\section{Etapa 1. Identificação da questão de pesquisa}

Para a formulação da pergunta de pesquisa utilizou-se a estratégia PCC - Participants (P), Concept (C) e Context (C).

Assim a pergunta de pesquisa formulada contemplou o PCC: Quais as evidências científicas, no contexto da APS (C), do uso das PICS (C) por idosos (P) para promoção, manutenção e recuperação da saúde, e para a prevenção de agravos?”

Os descritores controlados foram selecionados a partir dos Descritores em Ciências da Saúde (DeCS), Medical Subject Headings Section (MeSH), CINAHL Headings e EMTREE, são apresentados no Quadro 1.

Quadro 1. Descritores controlados.

\begin{tabular}{|l|l|}
\hline DeCS & $\begin{array}{l}\text { Atenção Primária à Saúde } \\
\text { Terapias Complementares } \\
\text { Idoso } \\
\text { Envelhecimento Saudável } \\
\text { Medicina Integrativa }\end{array}$ \\
\hline MeSH & $\begin{array}{l}\text { Primary Health Care. } \\
\text { Complementary Therapies } \\
\text { Aged } \\
\text { Healthy Aging } \\
\text { Integrative Medicine }\end{array}$ \\
\hline Cinahl & $\begin{array}{l}\text { Primary health care or primary care } \\
\text { Aged or elderly or senior or older people or geriatric } \\
\text { Complementary therapies or alternative therapies }\end{array}$ \\
\hline Emtree & $\begin{array}{l}\text { Aged } \\
\text { Primary health care } \\
\text { Alternative medicine }\end{array}$ \\
\hline
\end{tabular}

Fonte: Autores (2021)

Os termos alternativos para os DeCS são apresentados no Quadro 2. 
Quadro 2. Termos alternativos para os DeCS.

\begin{tabular}{|c|c|c|c|}
\hline Descritor em português & $\begin{array}{c}\text { Terapias Complementares } \\
(\text { Concept })\end{array}$ & $\begin{array}{c}\text { Atenção Primária à Saúde } \\
\text { (Context) }\end{array}$ & $\begin{array}{c}\text { Idoso } \\
\text { (Participants) }\end{array}$ \\
\hline Termos alternativos & $\begin{array}{l}\text { Magnetismo Vegetal } \\
\text { Medicina Alternativa } \\
\text { Medicina Complementar } \\
\text { Medicina Complementar e } \\
\text { Integrativa } \\
\text { Medicina Integrativa e } \\
\text { Complementar } \\
\text { Práticas Complementares e } \\
\text { Integrativas } \\
\text { Práticas Integrativas e } \\
\text { Complementares } \\
\text { Práticas de Saúde } \\
\text { Complementares e Integrativas } \\
\text { Práticas de Saúde Integrativas e } \\
\text { Complementares } \\
\text { Terapias Alternativas } \\
\text { Terapias Complementares e } \\
\text { Integrativas }\end{array}$ & $\begin{array}{l}\text { Atendimento Básico } \\
\text { Atendimento Primário } \\
\text { Atendimento Primário de Saúde } \\
\text { Atenção Básica } \\
\text { Atenção Básica à Saúde } \\
\text { Atenção Básica de Saúde } \\
\text { Atenção Primária } \\
\text { Atenção Primária de Saúde } \\
\text { Atenção Primária em Saúde } \\
\text { Cuidados de Saúde Primários } \\
\text { Cuidados Primários } \\
\text { Cuidados Primários à Saúde } \\
\text { Cuidados Primários de Saúde } \\
\text { Primeiro Nível de Assistência } \\
\text { Primeiro Nível de Atendimento } \\
\text { Primeiro Nível de Atenção } \\
\text { Primeiro Nível de Atenção à } \\
\text { Saúde } \\
\text { Primeiro Nível de Cuidado } \\
\text { Primeiro Nível de Cuidados }\end{array}$ & $\begin{array}{l}\text { Idosos } \\
\text { Pessoa de Idade } \\
\text { Pessoa Idosa } \\
\text { Pessoa de Idade } \\
\text { Pessoas Idosas } \\
\text { População Idosa }\end{array}$ \\
\hline
\end{tabular}

Fonte: Autores (2021).

\section{Etapa 2. Identificação dos estudos relevantes}

A busca dos estudos será realizada nas seguintes bases de dados: Medical Literature Analysis and Retrievel System Online (Medline) via PubMed da U.S. National Library of Medicine (NLM), Cumulative Index to Nursing and Allied Health Literature (CINAHL), Web of Science, Literatura Latino-Americana e do Caribe em Ciências da Saúde (LILACS); Scientific Electronic Library Online (Scielo), Base de Dados Bibliográfica sobre Cuidados de Salud en Iberoamérica (CUIDEN), Scopus e Embase. A busca na literatura cinzenta de materiais não indexados será conduzida por meio do Google Acadêmico.

Como os resultados serão obtidos em base de dados de material já publicado, assim não haverá necessidade de anuência de um Comitê de Ética em Pesquisa.

A estratégia de busca foi adaptada de acordo com as especificidades de cada base de dados, mantendo-se a similaridade da combinação dos descritores por meio dos operadores booleanos (OR e $A N D)$ conforme Quadro 3. 
Quadro 3. Estratégia de busca.

\begin{tabular}{|c|c|}
\hline BVS/BIREME & $\begin{array}{l}\text { primary health care AND complementary therapies AND aged AND (db:("MEDLINE" OR "LILACS" } \\
\text { OR "BDENF" OR "IBECS" OR "LIS" OR "CUMED" OR "MedCarib") }\end{array}$ \\
\hline Medline via PubMed & $\begin{array}{l}((((((\text { Primary Health Care"[Title/Abstract] }) \text { OR ("Primary Health Care"[MeSH Terms])) AND } \\
(\text { complementary therapies[Title/Abstract])) OR (complementary therapies[MeSH Terms])) OR } \\
\text { ("integrative medicine"[Title/Abstract])) OR ("integrative medicine"[MeSH Terms])) AND (aged) }\end{array}$ \\
\hline Cinahl & $\begin{array}{l}\text { ("primary health care" or "primary care") AND ("complementary therapies" or "alternative therapies") } \\
\text { AND (aged or elderly or senior or older people or geriatric) }\end{array}$ \\
\hline Web of Science & $\begin{array}{l}(((((\text { ALL }=(\text { Primary Health Care })) \text { OR } \\
\text { TS }=(\text { Primary Health Care })) \text { AND } \\
\text { ALL }=(\text { Complementar y Therapies })) \text { OR } \\
\text { TS }=(\text { Complementary Therapies })) \text { AND } \\
\text { ALL }=(\text { aged })) \text { AND } \\
\text { TS }=(\text { aged })\end{array}$ \\
\hline EMBASE & $\begin{array}{l}\text { (('primary health care' OR 'primary health care'/exp/mj) AND 'alternative medicine':ti,ab,kw OR } \\
\text { 'alternative medicine'/exp) AND aged:ti,ab,kw AND [2019-2021]/py }\end{array}$ \\
\hline SCOPUS & $\begin{array}{l}\text { ( TITLE-ABS-KEY ( \&quot;Primary } \\
\text { Health Care\&quot; ) AND TITLE-ABS- } \\
\text { KEY ( \&quot;Complementary } \\
\text { Therapies\&quot; ) AND TITLE-ABS- } \\
\text { KEY ( aged ) OR TITLE-ABS- } \\
\text { KEY ( healthy AND aging ) ) } \\
\end{array}$ \\
\hline CUIDEN & $\begin{array}{l}\text { ("Atención Primaria de Salud" OR "Terapias Complementarias") and Anciano OR ("Envejecimiento } \\
\text { Saludable") OR ("Medicina Integral") }\end{array}$ \\
\hline Google acadêmico & $\begin{array}{l}\text { (“Atenção Primária à Saúde" OR "Primary Health Care") AND ("Terapias Complementares" OR } \\
\text { "Complementary Therapies") AND (Idoso OR Aged) AND (Envelhecimento Saudável OR "Healthy } \\
\text { Aging”) OR (“Medicina Integrativa" OR "Integrative Medicine") }\end{array}$ \\
\hline
\end{tabular}

Fonte: Autores (2021).

Para a pesquisa serão incluídos artigos em português, espanhol e inglês, por serem línguas de conhecimento das pesquisadoras, não sendo definido limite temporal para permitir a obtenção de todos os dados possíveis sobre o tema. Também não será definido o limite do local de publicação, para realizar uma busca exaustiva e localizar as evidências nacionais e internacionais.

Na revisão de escopo serão aplicadas pesquisas originais, relatos de experiências, ensaios teóricos (ou reflexões teóricas), revisões narrativas e integrativas, manuais do Ministério da Saúde publicadas na íntegra em inglês, espanhol e português que versam sobre os benefícios do uso das PICS por idosos na APS no Brasil, independentemente do ano do estudo. Como critérios de exclusão foram definidos os seguintes itens: resenhas, cartas, estudos de caso, estudos em outro idioma não estabelecidos e artigos na íntegra não disponíveis gratuitamente e online

\section{Etapa 3. Seleção e avaliação inicial dos estudos}

Nessa etapa, a avaliação do título e do resumo de todos os estudos encontrados será efetuada, por duas pessoas independentes, fundamentada nos critérios de inclusão e exclusão determinados. Com isso, os estudos pertinentes serão selecionados para leitura na íntegra e posterior extração de dados.

\section{Etapa 4. Análise dos dados}

Os artigos selecionados na etapa anterior para leitura na íntegra terão os seguintes dados extraídos, conforme as variáveis apresentadas no Quadro 4. 
Quadro 4. Ficha de extração de dados da scoping review.

\begin{tabular}{|l|l|}
\hline Variável & Dados \\
\hline Tipo de estudo & Artigo, dissertação ou tese \\
\hline Ano de publicação & Ano em que a pesquisa foi publicada \\
\hline País de origem & País de condução do estudo \\
\hline Objetivo & Examinar o objetivo da pesquisa \\
\hline Tipo de pesquisa & De acordo com a descrição do autor \\
\hline Local & Local de realização do estudo \\
\hline Participantes do estudo & Quem foram os participantes do estudo \\
\hline Resultados encontrados & $\begin{array}{l}\text { Descrição das PICS utilizadas por idosos, no contexto da APS, para para a } \\
\text { promocão, manutenção e recuperação da saúde, e para a prevenção de } \\
\text { agravos }\end{array}$ \\
\hline Recomendações & \begin{tabular}{l} 
Detalhar as recomendações sugeridas pela pesquisa \\
\hline
\end{tabular} \\
\hline
\end{tabular}

Fonte: Autores (2021).

\section{Etapa 5. Agrupamento, síntese e apresentação dos dados}

A seleção das informações na etapa 4 permitirá uma maior compreensão e apresentação dos resultados por meio da estatística descritiva. Essa etapa será desenvolvida com base nas recomendações do guia internacional Preferred Reporting Items for Systematic and Meta-Analyses - Extension for Scoping Reviews (PRISMA-SrC) (Tricco et al., 2018). Os dados coletados serão reunidos em uma planilha que será formulada no Microsoft Excel 2010 e discutidos mediante a literatura pertinente.

\section{Considerações Finais}

Sugere-se que essa scoping review seja realizada com a intenção de fornecer um mapeamento da utilização das PICS por idosos, como método para a promoção da saúde, prevenção de agravos, além da manutenção e recuperação da saúde. As PICS na APS, porta de entrada do SUS possuem grande capacidade resolutiva e proporcionam o envelhecimento saudável e com qualidade dos idosos.

\section{Referências}

Almeida, C. R. F., Alcântara, J. T. \& Queiroz, T. A. (2021). Práticas integrativas e complementares em saúde: utilização e eficácia para melhoria da vida do idoso: integrative and complementary practices in health: use and effectiveness to improve life of the elderly. Brazilian Journal Of Development: Brazilian Journal of Development, 7(1), 1757-1765. https://www.brazilianjournals.com/index.php/BRJD/article/view/22736/18222.

Aromataris, E., \& Munn, Z. (2020). Joanna Briggs Institute Manual for Evidence Synthesis. JBI.

Brasil. (1988). Constituição de 1988. Constituição da República Federativa do Brasil: texto constitucional promulgado em 5 de outubro de 1988, com as alterações determinadas pelas Emendas Constitucionais de Revisão nos 1 a 6/94, pelas Emendas Constitucionais nos 1/92 a 91/2016 e pelo Decreto Legislativo no 186/2008. Brasília: Senado Federal, Coordenação de Edições Técnicas, 2016. 496 p

Brasil. (2006a). Ministério da Saúde. Política Nacional de Práticas Integrativas e Complementares no SUS. Secretaria de Atenção à Saúde, 92 p. https://bvsms.saude.gov.br/bvs/publicacoes/pnpic.pdf.

Brasil. (2006b). Ministério da Saúde. Gabinete do Ministro. Portaria $n^{\circ}$ 971, de 3 maio de 2006. Aprova a Política Nacional de Práticas Integrativas e Complementares (PNPIC) no Sistema Único de Saúde. Diário Oficial da União, Brasília, DF.(84),20-25. Seção. https://bvsms.saude.gov.br/bvs/saudelegis/gm/2006/prt0971_03_05_2006.html. 
Brasil. (2017a). Ministério da Saúde. Portaria de Consolidação no 02, Anexo XXII, de 28 de setembro de 2017. Aprova a Política Nacional de Atenção Básica. Brasília: Ministério da Saúde. https://bvsms.saude.gov.br/bvs/saudelegis/gm/2017/prc0002_03_10_2017.html.

Brasil. (2017b). Ministério da Saúde. Portaria no 849, de 27 de março de 2017. Inclui a Arteterapia, Ayurveda, Biodança, Dança Circular, Meditação, Musicoterapia, Naturopatia, Osteopatia, Quiropraxia, Reflexoterapia, Reiki, Shantala, Terapia Comunitária Integrativa e Yoga à Política Nacional de Práticas Integrativas e Complementares. Diário Oficial da $\quad 2017$. https://bvsms.saude.gov.br/bvs/saudelegis/gm/2017/prt0849_28_03_2017.html.

Brasil. (2018a). Ministério da Saúde. Secretaria de Vigilância em Saúde. Secretaria de Atenção à Saúde. Política Nacional de Promoção da Saúde: PNPS: Anexo I da Portaria de Consolidação $\mathrm{n}^{\circ}$ 2, de 28 de setembro de 2017, que consolida as normas sobre as políticas nacionais de saúde do SUS. Brasília: Ministério da Saúde, 2018. 40 p. https://bvsms.saude.gov.br/bvs/publicacoes/politica_nacional_promocao_saude.pdf.

Brasil. (2018b). Ministério da Saúde. Secretaria de Atenção à Saúde. Departamento de Atenção Básica. Política nacional de práticas integrativas e complementares no SUS: atitude de ampliação de acesso. Ministério da 96 Saúde, https://bvsms.saude.gov.br/bvs/publicacoes/politica_nacional_praticas_integrativas_complementares_2ed.pdf.

Brasil. (2018c). Ministério da Saúde. Secretaria de Atenção à Saúde. Departamento de Atenção Básica. Manual de implantação de serviços de práticas integrativas e complementares no SUS. da Saúde 56 p. http://189.28.128.100/dab/docs/portaldab/publicacoes/manual_implantacao_servicos_pics.pdf.

Brasil. (2021). Ministério da Saúde. Política Nacional de Práticas Integrativas e Complementares no SUS. Brasília: Ministério da Saúde. https://aps.saude.gov.br/ape/pics.

Cordeiro, L. \& Soares, C. B. (2019). Revisão de escopo: potencialidades para a síntese de metodologias utilizadas em pesquisa primária qualitativa. Boletim do Instituto de Saúde - BIS, 20 (2), 37-43. http://docs.bvsalud.org/biblioref/2019/10/1021863/bis-v20n2-sintese-de-evidencias-qualitativas-37-43.pdf>.

Giovanella, L. et al. (2019). De Alma-Ata a Astana. Atenção primária à saúde e sistemas universais de saúde: compromisso indissociável e direito humano fundamental. Cadernos de Saúde Pública, 35(3), e00012219. https://doi.org/10.1590/0102-311X00012219.

Habimorad, P. H. L. et al. (2020). Potencialidades e fragilidades de implantação da Política Nacional de Práticas Integrativas e Complementares. Ciênc Saúde Coletiva, 25(2), 395-405. http://www.scielo.br/scielo.php?script=sci_arttext\&pid=S1413-81232020000200395\&lng=en.

Moebus, R. L. N. \& Merhy, E. D. (2017). Genealogia da Política Nacional de Práticas Integrativas e Complementares. Saúde em Redes, 3(2): 145-152. http://revista.redeunida.org.br/ojs/index.php/rede-unida/article/view/887.

Marques, P. P., Francisco, P. M. S. B. B., Bacuru, A. G. M., Rodrigues, P. S., Malta, D. C. \& Barros, N. F. (2020). Uso de Práticas Integrativas e Complementares por idosos: pesquisa nacional de saúde 2013. Saúde em Debate, 44(126): 845-856. https://www.scielo.br/j/sdeb/a/KhF5fQSCKGWbzqg4j7kTQPP/?lang=pt.

Nascimento, L. C., Viegas, S. M. F, Menezes, C., Roquini, G. R. \& Santos, T. R. (2020). O SUS na vida dos brasileiros: assistência, acessibilidade e equidade no cotidiano de usuários da Atenção Primária à Saúde. Physis: Revista de Saúde Coletiva, 30(03), e300330. https://doi.org/10.1590/S0103-73312020300330.

Pinto, G. F. et al. (2020). Uso de práticas integrativas e complementares por idosos. Saúde e Pesquisa, 13 (2), 275-282. https://periodicos.unicesumar.edu.br/index.php/saudpesq/article/view/7563.

Silva, G. K. F., Sousa, I. M. C., Cabral, M. E. G. S, Bezerra, A. F. B. \& Guimarães, M. B. L. (2020). Política Nacional de Práticas Integrativas e Complementares: trajetória e desafios em 30 anos do SUS. Physis: Revista de Saúde Coletiva, 30(01), e300110. https://doi.org/10.1590/S010373312020300110 .

Silva, J. F., Fonseca, D. F., Silva J, Viegas, S.M. F. \& Lanza, F.M. (2019). O cotidiano de trabalho da estratégia saúde da família: entre o real e o ideal. Revista de Enfermagem do Centro-Oeste Mineiro. 9, e3488. http://seer.ufsj.edu.br/index.php/recom/article/view/3488/2276.

Sucharew, H. \& Macaluso, M. (2019). Methods for Research Evidence Synthesis: The Scoping Review Approach. Journal of Hospital Medicine, 14 (7): 416418. https://www.journalofhospitalmedicine.com/jhospmed/article/202729/hospital-medicine/methods-research-evidence-synthesis-scoping-review.

Tricco, A.C. et al. (2018) PRISMA Extension for Scoping Reviews (PRISMAScR): Checklist and Explanation. Ann Intern Med. 169, 467-473.

WHO. (2019). Word Health Organiztion. WHO Global Report on Traditional and Complementary Medicine. Geneva: World Health Organization. https://www.who.int/traditional-complementary-integrative-medicine/WhoGlobalReportOnTraditionalAndComplementaryMedicine2019.pdf. 